\title{
GESTÃO DE ESTOQUE POR MEIO DO MRP: UM ESTUDO DE CASO EM UMA METALÚRGICA DE MARINGÁ-PR
}

\section{INVENTORY MANAGEMENT WITH MRP: A CASE STUDY IN A METALLURGICAL OF MARINGÁ-PR}

\author{
Daiane Maria De Genaro Chiroli ${ }^{1}$; Karen de Oliveira Valério ${ }^{2}$ \\ ${ }^{1}$ Universidade Estadual de Maringá- UEM - Maringá/PR - Brasil \\ dmgenaro@hotmail.com \\ ${ }^{2}$ Universidade Estadual de Maringá- UEM - Maringá/PR - Brasil \\ karen.valerio@yahoo.com.br
}

\begin{abstract}
Resumo
No mercado atual onde os consumidores exigem cada vez mais qualidade e maior agilidade na entrega de seus fornecedores, possuir um adequado planejamento e programação da produção se torna mais que necessário, se torna essencial para a sobrevivência de uma empresa no mercado tão competitivo. Este trabalho aborda a implementação de um sistema de gestão de estoques por meio do Planejamento de Necessidades de Materiais em metalúrgica na cidade de Maringá, com a finalidade de diminuir os atrasos nas entregas das ordens de produção e realizar um planejamento das compras de matérias-primas para atender as demandas dos clientes sem atrasos. Através da análise da demanda mensal dos produtos e de sua organização em estrutura foi possível visualizar todos os componentes e matérias-primas necessárias para a sua produção. Esses dados serviram como base para o planejamento e a programação das compras dos materiais e da produção dos produtos, desde a liberação das ordens planejadas, recebimentos das ordens, controle do estoque, até o acompanhamento da demanda semanal do produto, contribuindo assim, para um processo produtivo sólido e otimizado. Foram obtidos como resultados a diminuição dos estoques de matérias-primas, o aumento da produtividade por meio da redução das paradas de produção devido à falta de materiais e a redução nos atrasos das entregas das ordens de produção.
\end{abstract}

Palavras-chave: gestão de estoques, planejamento de necessidades de materiais, MRP, produtividade.

\section{Introdução}

"A competitividade de um país depende da capacidade da sua indústria de inovar e melhorar" (PORTER, 1999, p.167). Assim como diz Porter a competitividade depende da melhoria de sua indústria, e um dos pontos importantes para se manter no mercado é gerenciar a produção de forma que melhore seus processos produtivos e reduza seus gastos, para isso ela tem que sempre inovar, buscar a melhor qualidade e o menor tempo através de uma melhoria contínua. 
"No cenário atual, clientes exigem um alto nível de serviço, pedidos mais frequentes, e trabalham no sentido de manter os níveis de estoque exatamente na medida de suas necessidades" (DIAS, 2010, p.110). Nesse contexto tem-se que os consumidores desejam sempre a melhor qualidade e a maior agilidade de seus fornecedores. Exercendo forte pressão sobre as indústrias.

Segundo Corrêa et al. (2001), um sistema de administração da produção deve ser capaz de apoiar o tomador de decisões logísticas, como planejar a necessidades futuras da produção, programar a produção para garantir que os recursos produtivos serão usados e ser capaz de oferecer os menores prazos possíveis aos clientes. Desta forma tem-se que planejar, programar e controlar a produção é de extrema importância estratégica para qualquer empresa que queira se manter no mercado. Para auxiliar as empresas a planejar e controlar seus recursos pode ser utilizado o Planejamento de Necessidades Materiais (MRP).

O MRP permite que as empresas calculem o número de materiais necessários para o funcionamento produtivo no momento determinado. Portanto o MRP verifica todos esses materiais e garante que sejam providenciados a tempo. O MRP é um sistema que ajuda as empresas a calcularem as quantidades de volume de materiais e o tempo necessário para adquiri-los (SLACK et al., 1999).

O presente trabalho será realizado para que com conceitos teóricos e uma boa análise da empresa, seja possível identificar uma solução para os problemas gerados pela má gestão do estoque, tornando adequada a função de um estoque. Onde não faltará mais matéria-prima e nenhuma produção será interrompida por falta dela. Para que se otimize os estoques sem acumulo ou excesso de matéria-prima. Dessa maneira a empresa poderá ser mais competitiva no mercado e melhorará o seu rendimento financeiro.

Este trabalho propõe mapear todos os suprimentos para a produção dos acabados, identificar o lead time produtivo, eliminar todos os custos desnecessários com acúmulos de estoque e reduzir os atrasos de entrega do produto acabado em uma metalúrgica na cidade de Maringá - Paraná. Deste modo, tem-se como objetivo geral estruturar um plano de gestão de estoques por meio do Planejamento de Necessidades de Materiais em uma metalúrgica na cidade de Maringá. A finalidade do estudo é fazer um melhor planejamento das compras de matéria-prima, para que não se tenha um estoque grande, mas o suficiente para atender as demandas dos clientes sem atrasos. Para isso será utilizado o conceito de PCP e de MRP. 


\section{Revisão de literatura}

\subsection{Planejamento e Controle da Produção}

Segundo Slack et al. (1999), planejar e controlar é garantir que a produção ocorra eficazmente e produza produtos e serviços como deve. Na quantidade adequada, no momento adequado e no nível de qualidade adequada.

Segundo Corrêa et al. (2001), planejar é entender como a situação presente e a visão futura influencia as decisões tomadas no presente para que possa atingir determinados objetivos futuros. É também projetar um futuro diferente do passado, por causas sobre as quais se tem controle.

"Em qualquer operação, o fornecimento de recursos não é infinito" (SLACK et al., 1999, p.231). Dessa maneira é possível ver que todas as operações possuem limitações, inclusive a de planejar e controlar. Ela pode ter limitações de custos, de capacidade, de tempo e de qualidade (SLACK et al., 1999).

Para ser lucrativa, uma empresa deve organizar todos os fatores, como maquinário, equipamentos e materiais, para fabricar os produtos certos no tempo certo, com o mais alto nível de qualidade e fazê-lo tão economicamente quanto possível (ARNOLD, 2012).

\subsection{Estoque}

De acordo com Slack et al. (2012, p278), estoque é "a acumulação armazenada de recursos materiais em um sistema de transformação." Estoques são materiais e suprimentos que uma empresa mantém para fornecer suprimentos no processo de fabricação (ARNOLD, 2012).

Segundo Corrêa et al. (2008), estoque são acúmulos de recursos materiais durante o processo de fabricação, e esses acúmulos proporcionam independência nesses processos. Acúmulos esses que podem ser para "o bem" ou para "o mal".

De acordo com Russomano (2000), o estoque é uma quantidade qualquer de material que seja armazenada, para uso futuro, por algum intervalo de tempo. Ele regula o ritmo entre os vários fluxos de material de uma empresa, realizando: cobertura das mudanças do mercado (aumento de preço); proteção contra incertezas (dificuldades na obtenção dos insumos) e compra econômica (comprar/produzir em grandes quantidades reduz as despesas).

Segundo Tubino (2000), os estoques possuem algumas funções principais, e são elas: garantir a independência entre etapas produtivas; permitir uma produção constante; possibilitar o uso de lotes econômicos; reduzir os lead times produtivos; como fator de segurança; para obter vantagens de preço. 


\subsection{Gestão de Estoque}

De acordo com Tubino (2000), a gestão do estoque é responsável pela definição do planejamento e controle dos níveis de estoques. Desde a matéria-prima ao produto acabado. E como os dois estoques, de matéria-prima e de acabados, dão apoio ou resultam da produção não podem ser controlados separadamente, devem ser coordenados (ARNOLD, 2012).

Segundo Dias (2010), um gestor de estoque deve conciliar os objetivos dos departamentos de compra, de produção, de venda e do financeiro, sem prejudicar a definição e a execução da politica de estoques da empresa.

A gestão de estoques deve controlar a disponibilidade total, ou seja, não deixar faltar matéria-prima ou produto acabado. E ela deve ter como objetivo a não falta do material sem ter que imobilizar demasiadamente os recursos financeiros da empresa (RUSSOMANO, 2000).

Segundo Slack et al. (1999) no gerenciamento e planejamento do estoque, os gerentes de produção estão envolvidos em três principais tipos de decisões: Quanto pedir; Quando pedir e Como controlar o sistema. A decisão de quanto pedir envolve diretamente os custos de manutenção e colocação do pedido. Já a decisão de quando pedir envolve a demanda, a necessidade do produto. E para controlar o sistema pode ser utilizado o modelo de controle de estoque por classificação $\mathrm{ABC}$.

Segundo Dias (2010), a curva ABC permite identificar aqueles itens que justificam atenção e tratamentos adequados em sua administração, por isso é um importante instrumento para a administração do estoque, para a definição de vendas, para o estabelecimento de prioridades e para a programação da produção. Após os itens terem sido ordenados por sua importância, as classes da curva $\mathrm{ABC}$ podem ser definidas.

Segundo Slack et al., (1999), normalmente a classe A são aqueles $20 \%$ de itens de alto valor. A classe B é representada por aqueles itens de valor médio, que representam $30 \%$ dos itens que representam $10 \%$ do valor total. $\mathrm{E}$ a classe $\mathrm{C}$ é representada por itens de baixo valor, menos importantes e não justificam muita atenção na administração do estoque.

“O MRP é um sistema que estabelece uma série de procedimentos e regras de decisão, de modo a atender às necessidades de produção numa sequência de tempo logicamente determinada para cada item componente do produto final" (DIAS, 2010, p.110).

De acordo com Dias (2010), trata-se de um sistema que define as quantidades necessárias e o tempo exato para a utilização na fabricação dos produtos finais. Possui como objetivos a garantia da disponibilidade dos materiais para o atendimento da produção e para a entrega aos clientes, preservar os inventários no nível mais baixo possível e planejar toda a atividade de manufatura, de suprimento e de programação das entregas. 
Segundo Slack et al., (1999), o MRP é um sistema que ajuda as empresas a fazer cálculos de volume e tempo da fabricação de cada produto. A Figura 1 ilustra todas as informações para processar o MRP, assim como alguns de seus resultados.

Figura 1- Esquema do planejamento de necessidades de materiais (MRP)

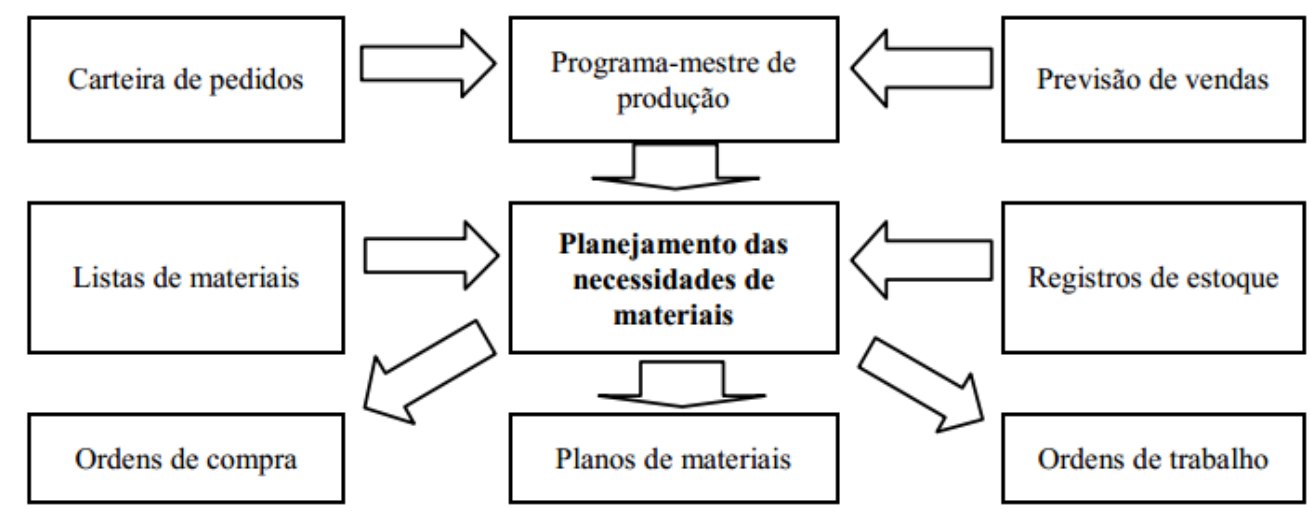

Fonte: SLACK et al., 1999, p 329

Conforme a Figura 1 todas as demais necessidades calculadas no processo MRP, são derivadas da carteira de pedidos e da previsão de demanda, sendo assim o MRP é um sistema de demanda dependente (SLACK et al., 1999).

A demanda dependente, assim como o próprio nome já diz, depende da demanda de outros itens, ou seja, o produto é dividido em vários componentes e esses componentes são divididos em níveis (ordens de produção), onde um nível é dependente do nível anterior (CHIROLI, 2014).

“(...) o programa MRP transforma a demanda do produto final em necessidades brutas para cada item ou componente" (DIAS, 2010, p.112).

De acordo com Dias (2010), todo o processo do planejamento do MRP inicia-se a partir das informações de quando e quanto o cliente deseja consumir. Esse processo de se orientar na demanda do cliente também é chamado de programa-mestre da produção (MSP), e é por ele que o MRP se orienta.

Segundo Slack et al., (1999), o programa-mestre de produção é constituído de registros dos tempos de cada produto final, das informações de demanda e de estoque disponível atual. E utilizando essas informações o estoque disponível é projetado á frente do tempo. E caso não exista estoque suficiente para atender a demanda futura, quantidades de pedidos são inseridas na linha do programa-mestre.

Segundo Dias (2010), relatórios e dados de saída são emitidos depois de completado o ciclo do programa MRP para serem utilizados no gerenciamento do processo logístico e de manufatura. 


\section{Metodologia}

Devido a atrasos de entrega de produtos acabados, caucionados pela falta de matéria-prima em uma metalúrgica em Maringá, notou-se a necessidade de um novo planejamento para a produção dos produtos e um melhor controle do estoque em relação à demanda dos clientes.

Desta maneira o presente trabalho visa mapear todos os itens que compõe os produtos acabados e controla-los de maneira que não ocasionem nenhum atraso na produção.

Segundo Silva e Menezes (2005) do ponto de vista de sua natureza essa pesquisa é considerada uma pesquisa aplicada, onde ela objetiva gerar conhecimentos para aplicação prática para a solução de problemas, envolvendo verdades.

Segundo Gil (2008) do ponto de vista dos objetivos trata-se de uma pesquisa exploratória, ou seja, proporciona maior familiaridade com o problema. Ela assume a forma de um estudo de caso, onde pode envolver levantamentos bibliográficos e informações retiradas de pessoas que estão envolvidas no problema. Quanto à abordagem, trata-se de uma pesquisa quantitativa, pois tudo pode ser quantificável. Irá utilizar o uso de recursos e técnicas estatísticas para classificar e analisar as informações coletadas.

Os passos identificados para a realização do trabalho estão exibidos na Figura 2.

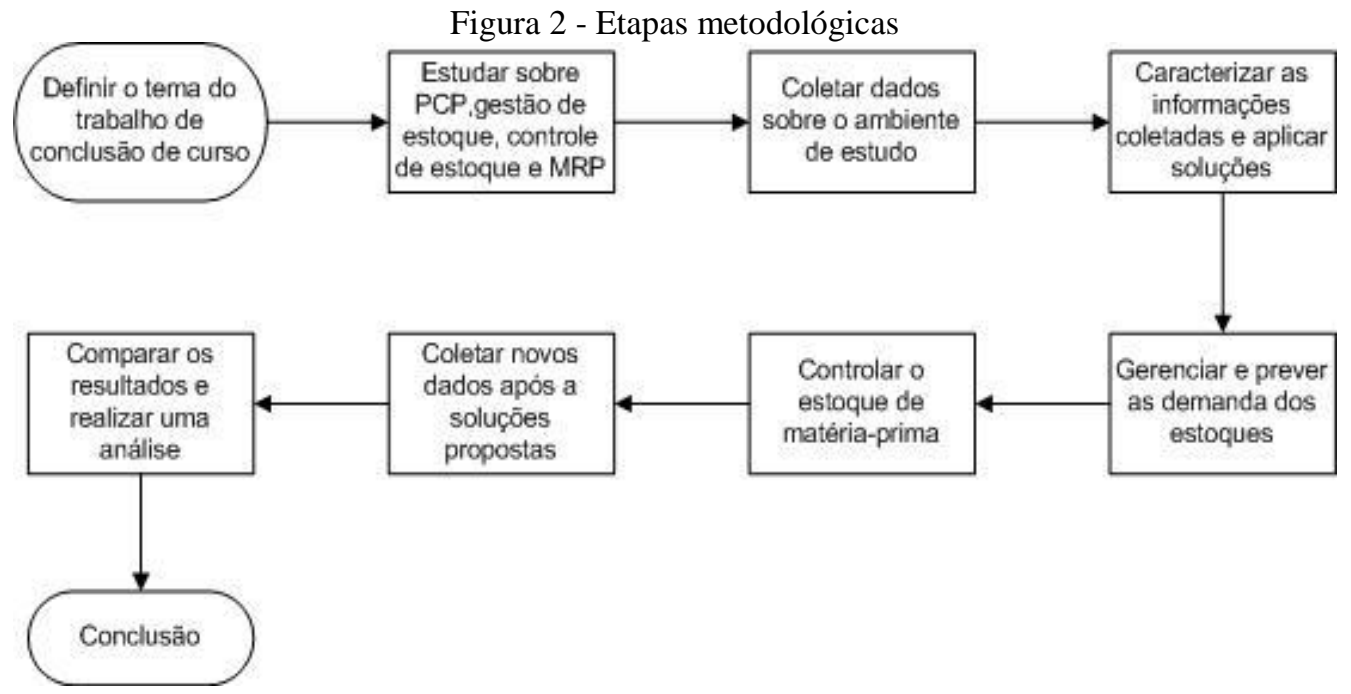

Conforme figura 2, os seguintes passos foram necessários para cumprir com o objetivo do presente trabalho:

1. Definição do tema abordado, por meio de análises dos processos de produção da empresa.

2. Levantamento literário sobre os conceitos relevantes, sendo eles: Planejamento e Controle da Produção, Estoque, Gestão de estoque e Material Resource Planning (MRP).

3. Coleta de todos os dados para a identificação do problema.

4. Caracterização dos dados coletados, analisando e aplicando soluções possíveis para cada problema encontrado, com base no método do MRP. 
5. Manter as soluções propostas em ação e gerencia-las para que não falte mais produtos acabados, por meio do gerenciamento e da previsão da demanda.

6. Monitorar os procedimentos aplicados nas compras e no estoque de matéria-prima, através do controle do estoque.

7. Analisar os resultados, realizando uma nova coleta de dados e a comparando com os resultados obtidos anteriormente.

8. Conclusão e apresentação do trabalho.

\section{Desenvolvimento}

\subsection{Caracterização da Empresa.}

O objeto de estudo deste trabalho é uma empresa do setor metal mecânico de pequeno porte fabricante de suportes para aparelhos eletroeletrônicos de áudio e vídeo, localiza-se na cidade de Maringá no estado do Paraná. A empresa iniciou suas atividades no ano de 2006, e desde então vem reformulando suas linhas de produtos para itens que sejam mais competitivos e atendam às necessidades dos clientes, necessidades estas que mudam à medida que os aparelhos eletroeletrônicos mudam ou evoluem. Dessa forma, as linhas de produtos da empresa passam por periódicas atualizações, assim como os produtos em catálogo são constantemente revistos a fim de tornar sua estrutura e processos de fabricação mais qualificados e competitivos.

Faz parte do catálogo de produtos da empresa uma série de linhas de suportes, as quais contam com uma média de 5 suportes cada, havendo portanto, cerca de 80 a 90 produtos em catálogo.

Atuam em média 25 colaboradores na empresa, que se dividem entre os setores de montagem, produção, prensagem e conformação, banho, pintura, expedição, almoxarifado, injeção de plástico, ferramentaria e administrativo. A Figura 3 ilustra o organograma de funções da empresa. 


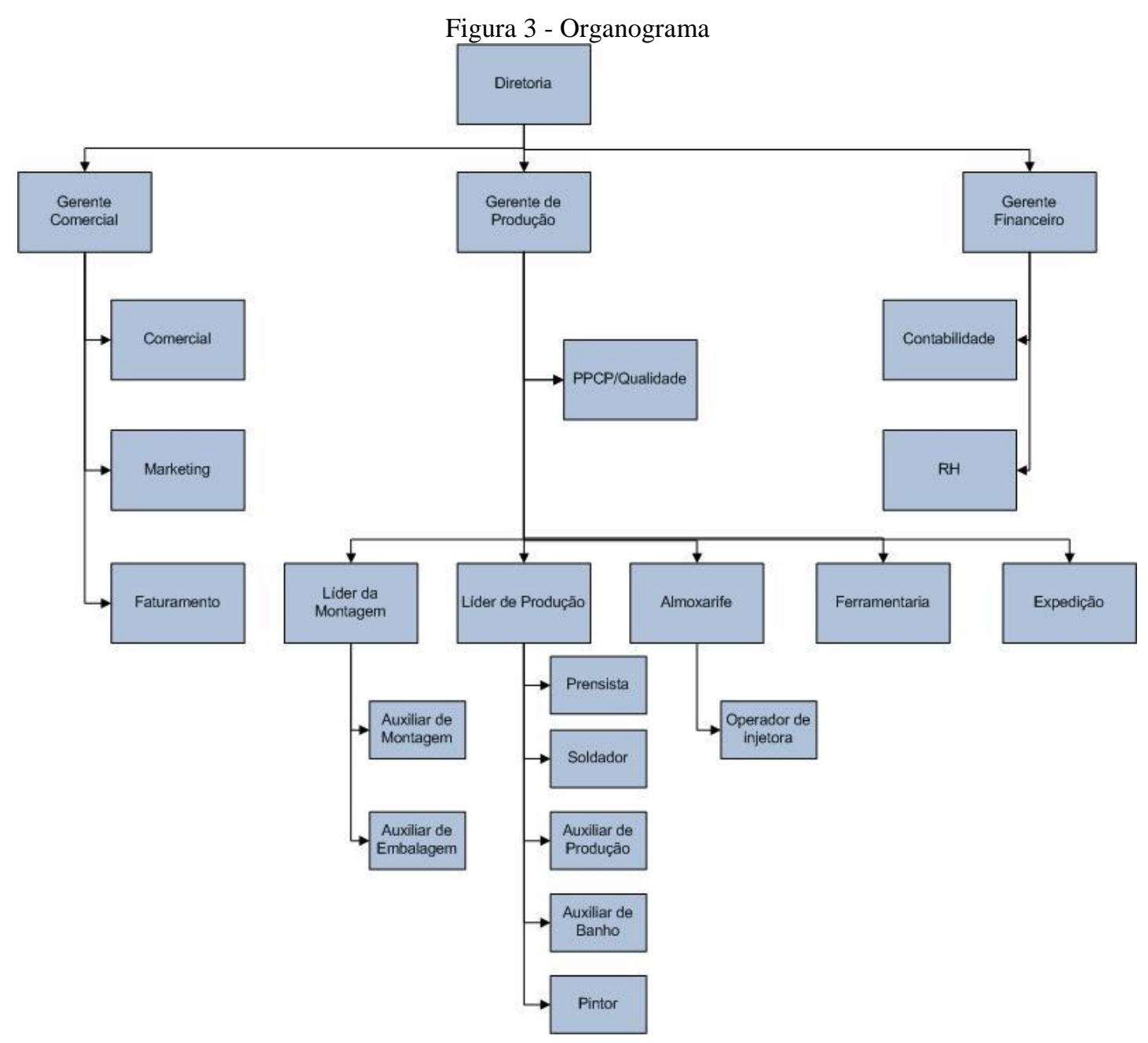

Há sete anos no mercado, a empresa disputa espaço com empresas atuantes no ramo há cerca de 30 anos, isso porque, apesar das falhas ainda existentes na execução e controle dos processos, a empresa destina esforços para que a não qualidade não atinja o consumidor final, os quais são atendidos sempre com eficácia. A empresa realiza também grandes investimentos na imagem de seus produtos dentro das lojas, o que facilita a competição com as grandes indústrias do setor.

A estratégia de mercado da empresa está na comercialização por meio de revendedores atuantes por alguns estados brasileiros para a venda de produtos para lojistas e distribuidoras. A empresa não conta com uma quantidade de representantes que considera ideal para a atual capacidade de produção, porém novas contratações levam tempo e a área comercial vem dedicando esforços na criação de estratégias para ampliação do sell in, fortalecendo a penetração dos produtos nos pontos de venda.

\subsection{Caracterização do Setor de Estudo}

Na empresa não há somente um setor exclusivamente responsável pelo controle do estoque. O que ocorre na prática é uma integração das atividades de alguns setores com o objetivo de manter 
os estoques em níveis adequados às necessidades. Esses setores são: PCP, Compras e Almoxarifado.

O PCP exerce a função de analisar as demandas de produtos acabados e as estratégias de promoção comercial e, a partir dessas análises, estipular os pontos de estoque mínimo e ponto de produção de cada item armazenado.

O almoxarifado é responsável por estar atento aos níveis de estoque e realizar solicitações de compra sempre que esses estiverem abaixo das quantidades previstas. O setor fica incumbido também de armazenar os produtos entregues pelos fornecedores, organizando os produtos estocados, mantendo-os separados por categorias, identificando os itens que não são mais usados na produção e dando as saídas de MP por meio do sistema ERP (Enterprise Resource Planning). Fica também sob responsabilidade do setor a realização de inventários periódicos, parciais ou totais, e atualização do ERP com os valores reais observados.

O setor de compras é responsável por analisar as solicitações de compras e realizar cotações com os principais fornecedores e, por fim, fechar os pedidos de acordo com as propostas mais convenientes.

\subsection{Processo Produtivo da Empresa}

A empresa possui diversas linhas de produtos e o processo de produção é o mesmo para todas elas. Ele se inicia com o departamento do PCP fazendo uma programação de ordens de produção $(\mathrm{OP})$, essa ordem de produção é entregue ao responsável pelo almoxarifado, onde será realizada a separação das matérias-primas e o encaminhamento dos itens para a produção, após a produção os componentes vão para o processo de montagem e então para a embalagem, após a embalagem o produto vai para o estoque da expedição. A Figura 4 ilustra esse fluxo dos processos.

Figura 4 - Fluxograma do processo produtivo

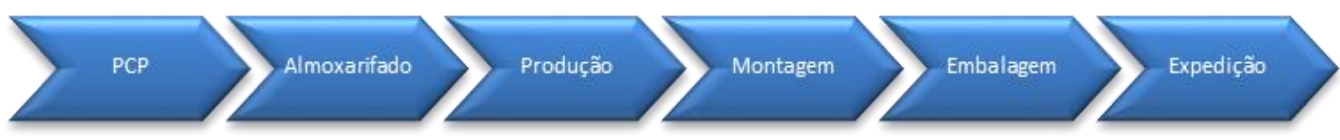

\subsection{Diagnóstico}

A empresa vem enfrentando dificuldades em atender seus clientes dentro do prazo estabelecido, devido à falta de alguns produtos. Falta essa que se dá devido aos atrasos que ocorrem durante a produção do mesmo. Com a forte concorrência do mercado a empresa se viu na necessidade de realizar um plano de ação para solucionar seus problemas de atrasos para então se tornar mais competitiva no mercado. 
Para conseguir entender exatamente qual era o problema foram feitas algumas análises. A primeira delas foi para descobrir quanto tempo uma ordem de produção levava para ser finalizada e para saber com qual frequência ela era finalizada fora do prazo estipulado. Para isso foi utilizada uma planilha do Microsoft excel onde contém as seguintes informações: número da OP; data de início da produção; data de entrega; quantidade; quantidade que falta (em casos que a entrada do produto é parcial); a referência do produto; seu status (concluído ou aberta); dia em que foi concluída a OP; lead-time planejado; lead-time real e o status 2 (caso tenha sido finalizada com atraso). A Figura 5 apresenta parte da planilha do controle do lead-time produtivo.

Figura 5 - Planilha de controle do lead-time produtivo

\begin{tabular}{|c|c|c|c|c|c|c|c|c|c|c|}
\hline & & & & & \multicolumn{2}{|c|}{ Indicadores do período } & $\begin{array}{c}\text { de } \\
01 / 05 / 2014\end{array}$ & $\begin{array}{l}\text { até } \\
30 / 05 / 2014\end{array}$ & & \\
\hline & & & & & \multicolumn{2}{|c|}{ OPs concluídas no período } & 51 & & & \\
\hline & & & & & \multicolumn{2}{|c|}{ OPs concluídas em atraso } & 30 & & & \\
\hline & & & & & \multicolumn{2}{|c|}{ Lead time da semana } & 9,8 & & & \\
\hline & & & & & \multicolumn{2}{|c|}{ OPs em aberto $(2014)^{*}$} & 25 & & & \\
\hline & & & & & \multicolumn{2}{|l|}{ Lead time (2014) } & 13,3 & & & \\
\hline & \multicolumn{3}{|c|}{$\begin{array}{c}\text { Controle Geral de OP } \\
\text { para a produção }\end{array}$} & & \multicolumn{6}{|c|}{ 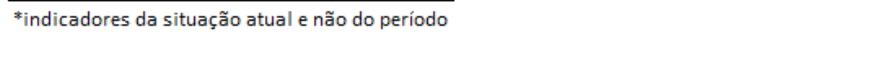 } \\
\hline № da OP & Data & Data & Qtdd & Qtdd Falta & Produto & Status & Hoje & & LT Real concl & Status2 \\
\hline$\nabla$ & $\mathbf{T}$ & Entrega $\rightarrow$ & $\nabla$ & $\nabla$ & $\checkmark$ & $\nabla$ & $\pi$ & Planejado $\nabla$ & $\checkmark$ & 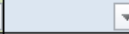 \\
\hline 1496 & 05/mai & $13 /$ mai & 12 & 12 & SLF-2040TTG-B & CONCLUÍDO & $06 / 05 / 2014$ & 7 & 2 & \\
\hline 1500 & 06/mai & $14 /$ mai & 30 & 1 & AR-4040T-B & CONCLUÍDO & $13 / 05 / 2014$ & 7 & 6 & \\
\hline 1502 & 07/mai & $15 /$ mai & 420 & 8 & MO-100-W & CONCLUÍDO & $10 / 06 / 2014$ & 7 & 25 & ATRASADO \\
\hline 1503 & $07 / \mathrm{mai}$ & $15 / \mathrm{mai}$ & 100 & 2 & LEX-730T-B & CONCLUÍDO & $27 / 05 / 2014$ & 7 & 15 & ATRASADO \\
\hline 1504 & 07/mai & $15 / \mathrm{mai}$ & 60 & 18 & TVD-1420-B & CONCLUÍDO & \begin{tabular}{|l|}
$16 / 05 / 2014$ \\
\end{tabular} & 7 & 8 & ATRASADO \\
\hline 1505 & 07/mai & $15 / \mathrm{mai}$ & 50 & 50 & TST-52-B & CONCLUÍDO & $23 / 05 / 2014$ & 7 & 13 & ATRASADO \\
\hline 1506 & 07/mai & $15 / \mathrm{mai}$ & 100 & 3 & ADP-01-B & CONCLUÍDO & $20 / 05 / 2014$ & 7 & 10 & ATRASADO \\
\hline 1507 & 07/mai & $15 /$ mai & 24 & 24 & STP-325-W & CONCLUÍDO & $22 / 05 / 2014$ & 7 & 12 & ATRASADO \\
\hline 1508 & 07/mai & $15 / \mathrm{mai}$ & 30 & 30 & AR-55T-B & CONCLUÍDO & 15/05/2014 & 7 & 7 & \\
\hline 1509 & 07/mai & $15 /$ mai & 99 & 9 & AR-730T-B & CONCLUÍDO & $22 / 05 / 2014$ & 7 & 12 & ATRASADO \\
\hline 1510 & 07/mai & $15 / \mathrm{mai}$ & 92 & 5 & AR-2040T-B & CONCLUÍDO & $23 / 05 / 2014$ & 7 & 13 & ATRASADO \\
\hline 1511 & 07/mai & $15 /$ mai & 50 & 50 & ADP-02-B & CONCLUÍDO & $16 / 05 / 2014$ & 7 & 8 & ATRASADO \\
\hline 1518 & 09/mai & $19 / \mathrm{mai}$ & 19 & 19 & SLF-2040TTG-B & CONCLUÍDO & $15 / 05 / 2014$ & 7 & 5 & \\
\hline 1519 & 09/mai & $19 /$ mai & 5 & 5 & SLF-2040TTG-W & CONCLUÍDO & $15 / 05 / 2014$ & 7 & 5 & \\
\hline 1520 & 09/mai & $19 /$ mai & 15 & 3 & ISM-2023-B & CONCLUÍDO & $22 / 05 / 2014$ & 7 & 10 & ATRASADO \\
\hline
\end{tabular}

É possível visualizar claramente na Figura 5 que a maioria das OP são finalizadas com atraso, desta maneira, fica evidente a existência de um problema na produção dos produtos e que precisa ser resolvido para que a empresa atinja o seu objetivo.

Para conseguir determinar qual era o problema dos atrasos foi distribuído para os supervisores da produção e da montagem fichas onde eles teriam que preencher, todas as vezes que houvesse parada na produção, com os motivos da parada. A Figura 6 apresenta ficha entregue para os supervisores.

Após um mês de fichas recolhidas foi realizada uma análise dos motivos que ocasionavam as paradas e em seguida foi elaborado um gráfico com eles, para tornar mais visível o problema. Na Figura 7 é possível verificar os motivos coletados e seu número de ocorrência. 
Figura 6 - Ficha de verificação de paradas na produção

\begin{tabular}{|c|c|c|c|}
\hline \multicolumn{5}{|c|}{ Relatório de controle de paradas de produção } \\
\hline Data & Matéria-Prima que faltou & Produto & Porque atrasou? \\
\hline & & & \\
\hline & & & \\
\hline & & & \\
\hline
\end{tabular}

Figura 7 - Gráfico para avaliação das paradas da produção

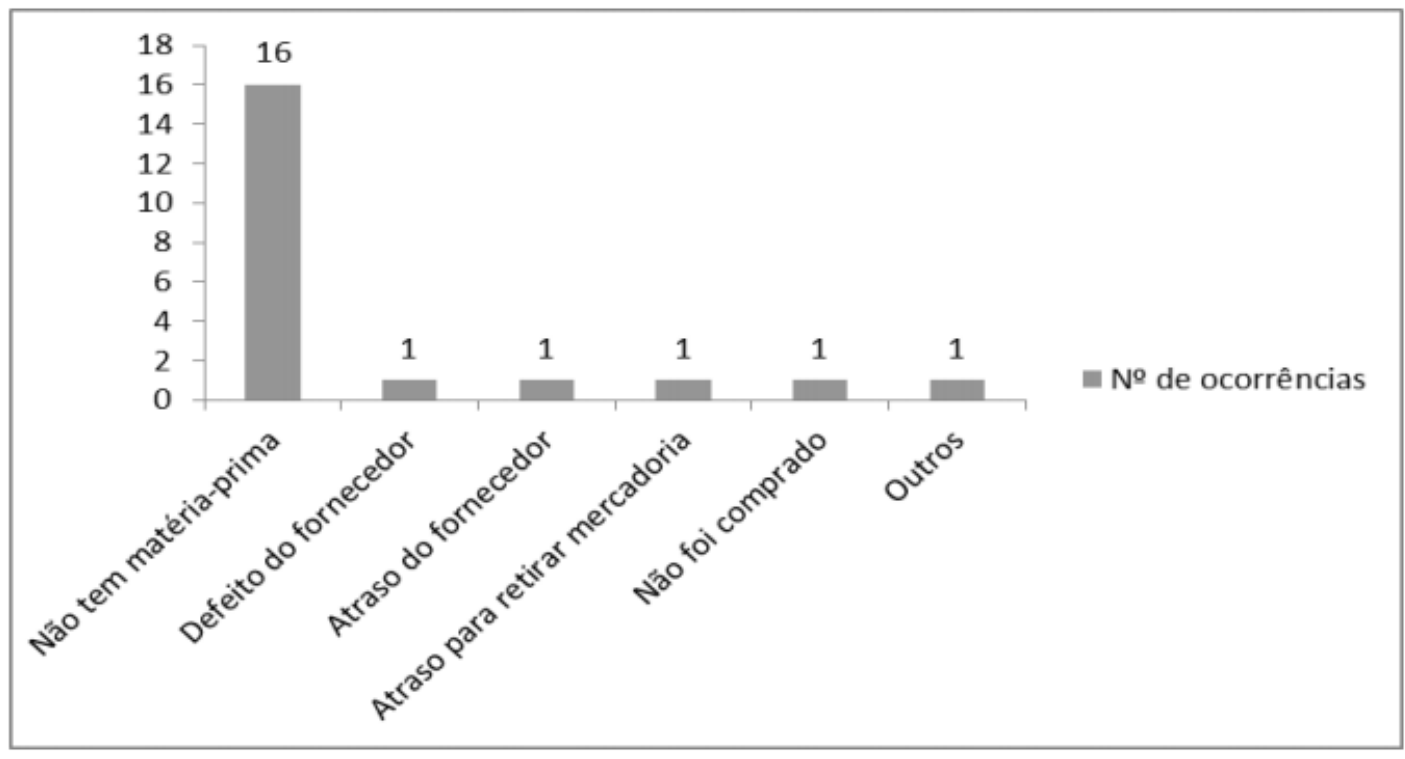

Analisando a Figura 7, obteve-se o maior causador das paradas na produção que geram os atrasos nas OP e na entrega do pedido ao cliente, a falta de matéria-prima. Dessa maneira, ao analisar o problema de forma mais profunda chegou-se a causa dos problemas, a inexistência de um planejamento das necessidades de materiais da indústria.

Através dessas informações decidiu-se que as ações que serão tomadas devem tratar a causa do problema, ou seja, a falta de um planejamento das necessidades materiais. Para isso será aplicado os conceitos do MRP, com o intuito de organizar e planejar toda a necessidade de material dentro da empresa.

\subsection{Implantação do MRP}

Para realizar o processo de implantação, o primeiro passo foi fazer uma classificação ABC dos produtos para identificar o produto que possui maior importância econômica para a empresa e então aplicar o processo primeiramente sobre ele, com a finalidade de obter um melhor planejamento das compras das matérias-primas e então conseguir atender a demanda mensal do produto sem nenhum tipo de atraso para o cliente final.

Na Figura 8 é possível identificar o produto com maior porcentagem de valor em cima do faturamento mensal da empresa. 
Figura 8 - Classificação ABC

\begin{tabular}{|c|c|c|c|c|c|}
\hline Ordem & Referência & Produto & $\begin{array}{l}\text { Qtde p/ } \\
\text { mês }\end{array}$ & $\% \mathrm{~S} /$ Fat. & Classificação \\
\hline 1 & AR-47T-B & SUP ARTIC PAR TILT TV LCD/LED ATE 47 - PRETO & 797 & 19,01 & $\mathrm{~A}$ \\
\hline 2 & SL3-1075-B & SUP FIXO PAREDE UNIV. TV LCD/LED 14-75 & 3.017 & 12,87 & B \\
\hline 3 & PSX-1075-B & SUP NYLON FIXO PARED UNIV TV LCD/LED $14-75-$ & 1.663 & 7,31 & $\mathrm{~B}$ \\
\hline 4 & AR-2040T-B & SUP BI-ARTI PAR TIL T TV LCD/LED 19 - 42 PRETO & 241 & 7,10 & $\mathrm{~B}$ \\
\hline 5 & AR2-730T-B & SUP BI- ARTIC PAR TILT TV LCD/LED ATE 40 PRETO & 247 & 6,00 & $\mathrm{~B}$ \\
\hline 6 & AR-730T-B & SUP ARTIC PAR TILT TV LCD/LED ATE 40 PRETO & 254 & 5,10 & $\mathrm{~B}$ \\
\hline 7 & AR-2040LT-B & SUP BI-ARTI PAR TIL T TV LCD/LED $19-42$ PRETO & 98 & 3,62 & $\mathrm{C}$ \\
\hline 8 & AR-55T-B & SUP ARTIC PAR TILT TV LCD/LED ATE 60" - & 80 & 3,61 & $\mathrm{C}$ \\
\hline 9 & MO-100-W & SUPORTE DE PAREDE P/ MICRO-ONDAS - & 421 & 2,45 & C \\
\hline 10 & $\mathrm{AR}-4040 \mathrm{~T}-\mathrm{B}$ & SUP BI-ARTI PAR TIL T TV LCD/LED $27-47$ PRETO & 45 & 2,04 & C \\
\hline 11 & STP-5075T-W & SUP. TETO P/ PROJETOR 50-75CM -TELESC. & 70 & 1,93 & C \\
\hline 12 & SL2-1070-B & SUP FIXO PAREDE UNIV. TV LCD/LED $14-70$ & 486 & 1,75 & C \\
\hline 13 & LEX-730T-B & SUP C/ TIL T FIXO PARED TV LED/LCD $40 \mathrm{C} / \mathrm{HASTE}$ & 183 & 1,73 & $\mathrm{C}$ \\
\hline 14 & SLM-4245-B & SUP. PAREDE SLIM VESA V420XH450 - PRETO & 133 & 1,63 & $\mathrm{C}$ \\
\hline 15 & ART-730T-B & SUP TETO TIL T TV LCD/LED ATE 40 PRETO & 57 & 1,19 & $\mathrm{C}$ \\
\hline 16 & STP-320-W & SUPORTE TETO PROJETOR 20CM -STANDARD & 54 & 1,06 & C \\
\hline 17 & ADP-01-B & SUPORTE PAREDE P/ DVD/BLU-RAY - PRETO & 156 & 0,97 & $\mathrm{C}$ \\
\hline 18 & SLF-4040TTG-B & SUP TETO GIRO TILT TV LCD/LED $27-47$ PRETO & 17 & 0,94 & C \\
\hline 19 & STP-312-W & SUPORTE TETO PROJETOR $12 \mathrm{CM}$ - STANDARD & 45 & 0,85 & $\mathrm{C}$ \\
\hline
\end{tabular}

Obteve-se então que o produto de maior influência no faturamento, apresentado na Figura 8, é o AR-47T-B. Sendo assim, nos próximos tópicos se apresentam os passos para a implantação do MRP sob a produção deste produto, onde será realizada a análise de sua demanda, a organização dos componentes do produto em níveis, o cálculo da necessidade de cada matéria-prima necessária e a programação para a obtenção desses materiais dentro do tempo correto e necessário, conforme segue.

\subsubsection{Análise da demanda}

Segundo Tubino (2000), a previsão da demanda é a base para o planejamento estratégico da produção. Ela possui uma função muito importante nos processos de planejamento dos sistemas de produção, pois permite que os administradores destes sistemas antevejam o futuro e planejem de forma adequada suas ações.

Ainda na Figura 8 é possível identificar que a saída mensal do AR-47T-B são de 797 peças por mês. Saber sobre a demanda dos itens é um elemento essencial para se obter o correto planejamento das necessidades dos materiais (CHIROLI, 2014). Neste estudo de caso temos uma demanda dependente, portanto é necessária a organização do produto em estrutura para se definir a quantidade necessária de cada componente à produção para cada unidade do produto.

\subsubsection{Organização do produto}

A organização do produto é realizada através de uma "explosão" de necessidades brutas de materiais, ou seja, é representar o produto através de uma estrutura onde é possível a visualização de todos os itens que são necessários para a fabricação do mesmo. Essa representação de estrutura do produto auxilia nas respostas de duas perguntas fundamentais do sistema de produção: o que e 
quanto produzir e comprar (CORRÊA et al., 2008). Na Figura 9 é apresentada a estrutura do produto em níveis, onde é possível visualizar todos os componentes utilizados para a produção do AR-47T-B.

Conforme mostra a Figura 9 o AR-47T-B possui três níveis em sua estrutura. Desta maneira é possível saber quais itens são necessários para a produção do suporte. Assim em seguida temos o Quadro 1 que lista todas as quantidades dos itens necessários para a produção.

Quadro 1: Lista de Materiais

\begin{tabular}{|c|c|c|c|}
\hline ITEM & QUANTIDADE & ITEM & QUANTIDADE \\
\hline Chapa de ferro & 1 unidade & $\mathrm{C}$ & 1 unidade \\
\hline U fêmea & 1 unidade & Vareta & 4 unidades \\
\hline Tubo oblongo $20 \mathrm{~cm}$ & 1 unidade & Cantoneira direita & 1 unidade \\
\hline Tubinho $41 \mathrm{~mm}$ & 1 unidade & $\begin{array}{l}\text { Cantoneira } \\
\text { esquerda }\end{array}$ & 1 unidade \\
\hline Tubinho $70 \mathrm{~mm}$ & 1 unidade & Kit de parafusos & 1 unidade \\
\hline Chapa de ferro $3 \mathrm{~mm}$ & 1 unidade & $\begin{array}{c}\text { Berço para } \\
\text { embalagem }\end{array}$ & 1 unidade \\
\hline U macho & 1 unidade & & \\
\hline
\end{tabular}

Através da análise dos dados do Quadro 1 é possível calcular a demanda mensal dos materiais que compõe o produto.

Figura 9 - Estrutura em níveis do AR-47T-B

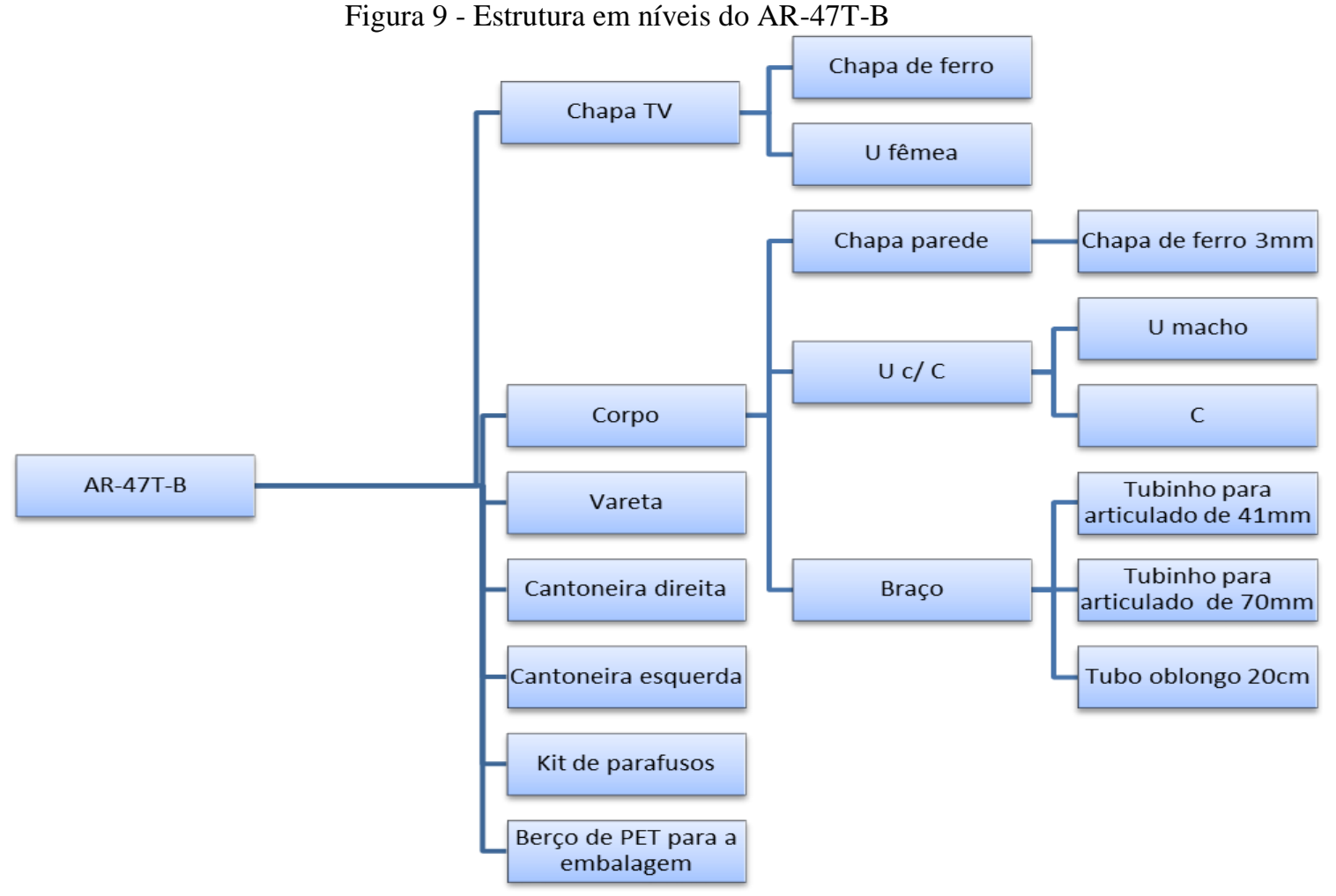




\subsubsection{Cálculo das necessidades dos materiais}

Segundo Corrêa et al. (2001), esse cálculo significa a quantidade total de componentes que necessita estar disponível para a fabricação das quantidades necessárias de produtos. No Quadro 2, podemos observar o quanto de cada componente será necessário para um mês de produção do AR47T-B e se ele é produzido na empresa ou comprado.

Quadro 2: Tabela de Necessidades de Materiais

\begin{tabular}{|l|c|l|l|}
\hline ITEM & QUANTIDADE & $\begin{array}{l}\text { QUANTIDADE } \\
\text { MENSAL }\end{array}$ & $\begin{array}{l}\text { COMPRADO } \\
\text { PRODUZIDO }\end{array}$ \\
\hline Chapa de ferro & 1 unidade & 797 unidades & Comprado \\
\hline U fêmea & 1 unidade & 797 unidades & Comprado \\
\hline Tubo oblongo $20 \mathrm{~cm}$ & 1 unidade & 797 unidades & Produzido \\
\hline Tubinho $41 \mathrm{~mm}$ & 1 unidade & 797 unidades & Produzido \\
\hline Tubinho $70 \mathrm{~mm}$ & 1 unidade & 797 unidades & Produzido \\
\hline Chapa de ferro $3 \mathrm{~mm}$ & 1 unidade & 797 unidades & Comprado \\
\hline U macho & 1 unidade & 797 unidades & Comprado \\
\hline C & 1 unidade & 797 unidades & Comprado \\
\hline Vareta & 4 unidades & 3188 unidades & Comprado \\
\hline Cantoneira direita & 1 unidade & 797 unidades & Comprado \\
\hline Cantoneira esquerda & 1 unidade & 797 unidades & Comprado \\
\hline Kit de parafusos & 1 unidade & 797 unidades & Produzido \\
\hline Berço para embalagem & 1 unidade & 797 unidades & Produzido \\
\hline
\end{tabular}

Fonte: Sistema ERP da empresa

No Quadro 2, temos a identificação de quantos itens serão necessários para atender a demanda mensal e temos também a classificação dos itens que são comprados ou produzidos, portanto aqueles que são produzidos na empresa possuem matérias-primas em sua estrutura que precisam ser compradas e desta forma é apresentado na Figura 10, na Figura 11 e na Figura 12 as estruturas dos componentes que são produzidos na empresa.

Figura 10 - Estrutura em níveis do kit de parafusos

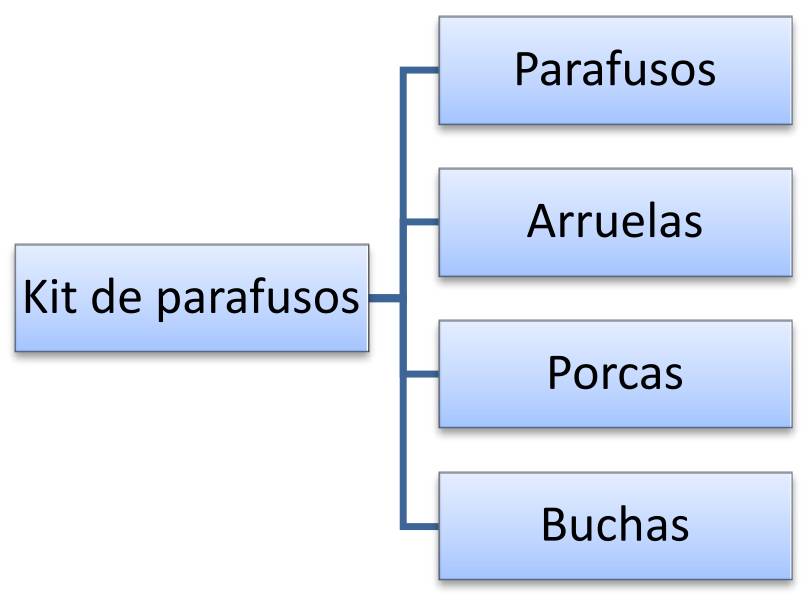


Figura 11 - Estrutura em níveis dos tubos

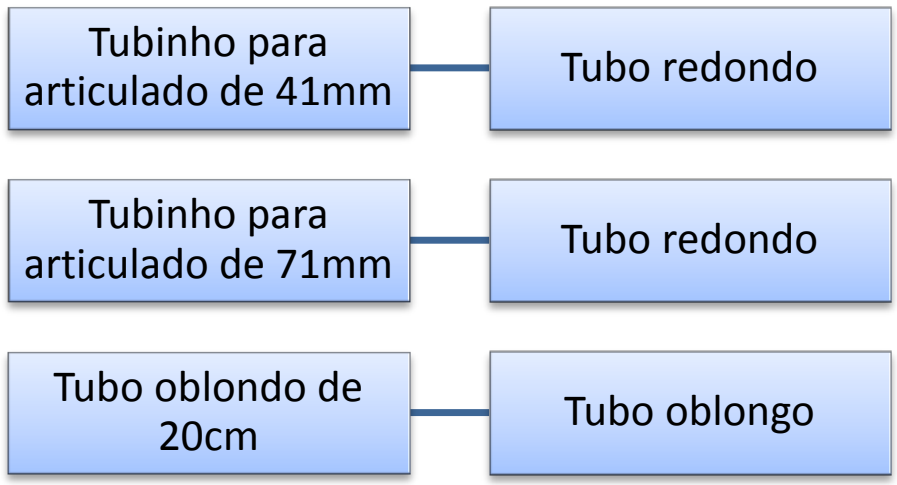

Figura 12 - Estrutura em níveis do berço de embalagem

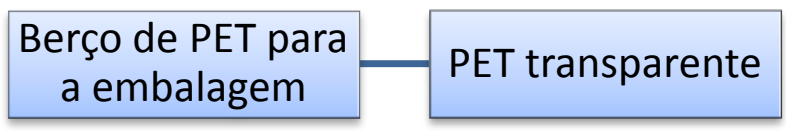

Através das estruturas dos componentes que são produzidos na empresa é possível identificar as matérias-primas necessárias para a produção deles. Desta maneira no Quadro 3 é apresentada as quantidades necessárias mensalmente dos itens identificados nas estruturas dos componentes.

Quadro 3: Tabela de Necessidades de Materiais dos Componentes

\begin{tabular}{|l|c|c|}
\hline ITEM & QUANTIDADE & $\begin{array}{l}\text { QUANTIDADE } \\
\text { MENSAL }\end{array}$ \\
\hline Tubo oblongo & 20 centímetro & 159,4 metros \\
\hline Tubo redondo & 112 milímetro & 89,27 metros \\
\hline Parafusos & 28 unidades & 22316 unidades \\
\hline Arruelas & 4 unidades & 3188 unidades \\
\hline Porcas & 8 unidades & 6376 unidades \\
\hline Buchas & 4 unidades & 3188 unidades \\
\hline PET transparente & $0,152 \mathrm{~kg}$ & $121,15 \mathrm{~kg}$ \\
\hline
\end{tabular}

Fonte: Sistema ERP da empresa

Como se pode observar nas Tabela 3 e Tabela 4, por meio da demanda foi possível calcular a necessidade mensal de cada matéria-prima do AR-47T-B. E com a informação de quantos itens serão necessários produzir e quantos serão necessários comprar podemos agora calcular o seu tempo de produção e de obtenção.

\subsubsection{Tempo necessário de obtenção dos materiais}

Com base nas informações de controle do lead-time de compras obteve-se o lead-time de cada fornecedor e através desta informação foi possível a geração do Quadro 4 onde é apresentado o tempo de obtenção de cada matéria-prima. 
Quadro 4: Tabela dos tempos de obtenção dos itens necessários

\begin{tabular}{|l|l|l|l|l|l|}
\hline Item & $\begin{array}{l}\text { Tempo de } \\
\text { obtenção }\end{array}$ & Fornecedor & Item & $\begin{array}{l}\text { Tempo de } \\
\text { obtenção }\end{array}$ & Fornecedor \\
\hline Chapa de ferro & 7 dias & 4 & Cantoneira direita & 7 dias & 4 \\
\hline $\begin{array}{l}\text { U fêmea } \\
\text { Tubo oblongo }\end{array}$ & 4 dias & 1 & Cantoneira esquerda & 7 dias & 4 \\
$\begin{array}{l}\text { Tubo redondo } \\
\text { Chapa de ferro }\end{array}$ & 4 dias & 3 & Parafusos & 16 dias & 5 \\
\hline $\begin{array}{l}\text { 3mm dias } \\
\text { U macho }\end{array}$ & 4 & Arruelas & 16 dias & 5 \\
\hline C dias & 1 & Porcas & 16 dias & 5 \\
\hline Vareta & 4 dias & 1 & Buchas & 16 dias & 5 \\
\hline
\end{tabular}

Fonte: Sistema ERP da empresa

Agora através dos dados demonstrados em todas as tabelas é possível iniciar o registro básico do MRP.

\subsubsection{Registro básico do MRP}

O registro básico do MRP disponibilizará informações sobre as quantidades e os prazos que deverão ser efetuadas as ordens de compra ou de produção (CHIROLI, 2014).

No Quadro 5 será apresentado o registro básico do produto AR-47T-B, sabendo que possui em estoque 55 unidades e que sua demanda semanal é de aproximadamente 200 peças.

Quadro 5: Registro básico AR-47T-B

\begin{tabular}{|c|c|c|c|c|c|c|}
\hline \multirow{2}{*}{ AR-47T-B } & \multicolumn{2}{|l|}{ Períodos (semanas) } & 1 & 2 & 3 & 4 \\
\hline & \multicolumn{2}{|l|}{ Necessidades brutas } & 200 & 200 & 200 & 200 \\
\hline \multirow{2}{*}{$\begin{array}{l}\text { Lead-time = } \\
1 \text { semana }\end{array}$} & \multicolumn{2}{|c|}{ Recebimentos programados } & & & & \\
\hline & Estoque projetado & 55 & 5 & 5 & 50 & 0 \\
\hline \multirow{2}{*}{$\begin{array}{l}\text { Lote minimo }= \\
150 \text { unidades }\end{array}$} & \multicolumn{2}{|c|}{ Recebimento de ordens planejadas } & 150 & 200 & 245 & 150 \\
\hline & \multicolumn{2}{|c|}{ Liberação de ordens planejadas } & 200 & 245 & 150 & \\
\hline
\end{tabular}

Fonte: Elaborado pelos autores

Trabalhando com um lote mínimo de produção de 150 peças, o Quadro 5 nos apresenta a programação da produção que deve ser feita para que nenhum pedido saia com atraso. Foram realizados os registros básicos de compras de todas as matérias-primas do AR-47T-B, como todas seguem a mesma lógica, será apenas demonstrado de algumas delas, conforme Quadros 6-10.

Quadro 6: Registro básico da chapa de ferro do AR-47T-B

\begin{tabular}{|c|c|c|c|c|c|c|}
\hline \multirow{2}{*}{ Chapa de ferro } & \multicolumn{2}{|l|}{ Períodos (semanas) } & 1 & 2 & 3 & 4 \\
\hline & \multicolumn{2}{|l|}{ Necessidades brutas } & 200 & 200 & 200 & 200 \\
\hline \multirow{2}{*}{$\begin{array}{l}\text { Lote minimo }=200 \\
\text { unidades }\end{array}$} & \multicolumn{2}{|c|}{ Recebimentos programados } & 250 & & & \\
\hline & Estoque projetado & 150 & 200 & 0 & 0 & 0 \\
\hline \multirow{2}{*}{$\begin{array}{l}\text { Lead-time } \\
\text { semana }\end{array}$} & \multicolumn{2}{|c|}{ Recebimento de ordens planejadas } & & & 200 & 200 \\
\hline & \multicolumn{2}{|c|}{ Liberação de ordens planejadas } & & 200 & 200 & \\
\hline
\end{tabular}


Quadro 7: Registro básico da chapa de ferro de 3mm do AR-47T-B

\begin{tabular}{|c|c|c|c|c|c|c|}
\hline \multirow{2}{*}{$\begin{array}{l}\text { Chapa de ferro } \\
3 \mathrm{~mm}\end{array}$} & \multicolumn{2}{|l|}{ Períodos (semanas) } & 1 & 2 & 3 & 4 \\
\hline & \multicolumn{2}{|l|}{ Necessidades brutas } & 200 & 200 & 200 & 200 \\
\hline \multirow{2}{*}{$\begin{array}{l}\text { Lote } \operatorname{minimo}=200 \\
\text { unidades }\end{array}$} & \multicolumn{2}{|c|}{ Recebimentos programados } & 150 & & & \\
\hline & Estoque projetado & 125 & 75 & 75 & 75 & 75 \\
\hline \multirow{2}{*}{$\begin{array}{l}\text { Lead-time } \\
\text { semana }\end{array}$} & \multicolumn{2}{|c|}{ Recebimento de ordens planejadas } & & 200 & 200 & 200 \\
\hline & \multicolumn{2}{|c|}{ Liberação de ordens planejadas } & 200 & 200 & 200 & \\
\hline
\end{tabular}

Fonte: Elaborado pelos autores

Quadro 8: Registro básico da cantoneira direita do AR-47T-B

\begin{tabular}{|c|c|c|c|c|c|c|}
\hline \multirow{2}{*}{ Cantoneira direita } & \multicolumn{2}{|l|}{ Períodos (semanas) } & 1 & 2 & 3 & 4 \\
\hline & \multicolumn{2}{|l|}{ Necessidades brutas } & 200 & 200 & 200 & 200 \\
\hline \multirow{2}{*}{$\begin{array}{l}\text { Lote minimo }=200 \\
\text { unidades }\end{array}$} & \multicolumn{2}{|c|}{ Recebimentos programados } & 250 & & & \\
\hline & Estoque projetado & 89 & 139 & 139 & 139 & 139 \\
\hline \multirow{2}{*}{$\begin{array}{l}\text { Lead-time }=1 \\
\text { semana }\end{array}$} & \multicolumn{2}{|c|}{ Recebimento de ordens planejadas } & & 200 & 200 & 200 \\
\hline & \multicolumn{2}{|c|}{ Liberação de ordens planejadas } & 200 & 200 & 200 & \\
\hline
\end{tabular}

Fonte: Elaborado pelos autores

Quadro 9: Registro básico da cantoneira esquerda do AR-47T-B

\begin{tabular}{|c|c|c|c|c|c|c|}
\hline \multirow{2}{*}{$\begin{array}{l}\text { Cantoneira } \\
\text { esquerda }\end{array}$} & \multicolumn{2}{|l|}{ Períodos (semanas) } & 1 & 2 & 3 & 4 \\
\hline & \multicolumn{2}{|l|}{ Necessidades brutas } & 200 & 200 & 200 & 200 \\
\hline \multirow{2}{*}{$\begin{array}{l}\text { Lote minimo }=200 \\
\text { unidades }\end{array}$} & \multicolumn{2}{|c|}{ Recebimentos programados } & 250 & & & \\
\hline & Estoque projetado & 89 & 139 & 139 & 139 & 139 \\
\hline \multirow{2}{*}{$\begin{array}{l}\text { Lead-time }=1 \\
\text { semana }\end{array}$} & \multicolumn{2}{|c|}{ Recebimento de ordens planejadas } & & 200 & 200 & 200 \\
\hline & \multicolumn{2}{|c|}{ Liberação de ordens planejadas } & 200 & 200 & 200 & \\
\hline
\end{tabular}

Fonte: Elaborado pelos autores

Quadro 10: Registro básico do U fêmea do AR-47T-B

\begin{tabular}{|c|c|c|c|c|c|c|}
\hline \multirow{2}{*}{ U fêmea } & \multicolumn{2}{|l|}{ Períodos (semanas) } & 1 & 2 & 3 & 4 \\
\hline & \multicolumn{2}{|l|}{ Necessidades brutas } & 200 & 200 & 200 & 200 \\
\hline \multirow{2}{*}{$\begin{array}{l}\text { Lote } \operatorname{minimo}=200 \\
\text { unidades }\end{array}$} & \multicolumn{2}{|c|}{ Recebimentos programados } & 150 & & & \\
\hline & Estoque projetado & 120 & 70 & 70 & 70 & 70 \\
\hline \multirow{2}{*}{$\begin{array}{l}\text { Lead-time }=1 \\
\text { semana }\end{array}$} & \multicolumn{2}{|c|}{ Recebimento de ordens planejadas } & & 200 & 200 & 200 \\
\hline & \multicolumn{2}{|c|}{ Liberação de ordens planejadas } & 200 & 200 & 200 & \\
\hline
\end{tabular}

Fonte: Elaborado pelos autores

Através das informações fornecidas pelos registros básicos dos componentes do AR-47T-B foi possível programar todas as compras, portanto o conceito do MRP pode ser aplicado para todos os produtos da empresa, desta maneira todas as compras serão programadas e planejadas e então pôr 
fim a empresa eliminará todos os problemas de atraso na entrega do produto e atrasos na produção devido a falta de matéria-prima.

\section{Discussão dos resultados}

Após a análise dos resultados é possível visualizar a importância de um bom planejamento e programação da produção e das compras de materiais e o quanto ela influencia na produtividade dos funcionários e nos processos de produção.

Por meio da classificação $\mathrm{ABC}$ foi identificado o produto com a maior importância econômica da empresa, e então em cima dele foi implantado o método MRP.

Primeiramente foi analisada a demanda mensal do produto, que permitiu o planejamento futuro das necessidades dos materiais. Em seguida, o produto, foi organizado em estrutura de níveis, tornando possível a visualização de todas as matérias-primas utilizadas para a sua produção.

Foi então calculada a necessidade mensal de cada matéria-prima com base na demanda mensal do produto e então foi calculado o seu tempo de obtenção, com base nos lead-time de entrega dos fornecedores.

Através dos tempos de obtenção e da demanda mensal de cada matéria-prima foi por fim realizada a programação da produção do produto e a programação de compra de cada matéria-prima que pertence a sua estrutura.

Por meio da programação da produção e das compras foi possível reduzir os estoques de matéria-prima, praticamente eliminar as faltas de materiais e por fim reduzir os atrasos nas entregas das ordens de produção.

\section{Conclusão}

O estudo de caso mostrou a importância de se ter um planejamento e uma programação das compras de matéria-prima em uma indústria, proporcionando melhor clareza nas tomadas de decisões, conciliando a demanda dos produtos com o tempo de compra e produção aproveitando, desta maneira, melhor os recursos e eliminando desperdícios.

Para a realização deste trabalho a revisão bibliográfica foi de extrema importância para a compreensão do MRP. Foram coletados dados e informações sobre os problemas de atrasos de modo que se tornou possível diagnosticar os reais problemas da produção e então propor a implantação do método do MRP.

Por meio da análise dos dados antes e após a implantação do método, fica claro que o objetivo principal de reduzir o atraso na entrega dos produtos foi alcançado, além disso, com a implantação foi possível melhorar a comunicação entre o departamento de compras e o PCP, dar maior agilidade ao fluxo de materiais e informações nos processos produtivos e reduzir 
desperdícios. Portanto, diante do que foi apresentado, conclui-se que a implantação do método do MRP foi de grande importância, contribuindo para o desenvolvimento e a otimização dos produtivos e administrativos através de um melhor planejamento da produção, sem dispor de grandes investimentos alcançou uma melhor satisfação dos clientes e fornecedores, tornando assim a empresa mais competitiva no mercado atual.

A maior dificuldade encontrada foi mostrar a importância e a necessidade da implantação do método para o responsável do departamento de compras. Por se tratar de uma ferramenta desconhecida, para ele, que iria modificar o seu modo de trabalho, foi necessário tempo e muitas reuniões para o esclarecimento da necessidade para então dar-se início a implantação.

Outra dificuldade que ocorreu foi a falta de um banco de dados onde relatasse os motivos de tantos atrasos, o sistema fornece apenas o status do pedido ou da ordem de produção mas não fornece o porquê de estar com atraso, e para solucionar esse problema foi necessário criar um banco de dados na ferramenta Microsoft Excel.

Como propostas de melhorias futuras, recomenda-se:

- Criar uma forma de programar as compras de matérias-primas com os fornecedores, ou seja, passar antecipadamente a ordem de compra no início do ano e conforme o passar do tempo o fornecedor ir entregando a quantidade mensal necessária;

- Criar indicadores para a avaliação do método de gestão de estoque implantado;

- Criar uma política de manutenção preventiva de todas as máquinas.

\begin{abstract}
In today's market where consumers increasingly demand quality and greater flexibility in the delivery of its suppliers have proper planning and production scheduling becomes more than necessary, it is essential for the survival of a company in such a competitive market. This paper discusses the implementation of an inventory management system via the Material Requirements Planning in metallurgy in the city of Maringa, in order to reduce delays in deliveries of the production orders and carry out a plan of purchases of raw materials to meet customer demands without delay. Through the monthly demand analysis of products and its organization structure it was possible to visualize all the components and raw materials needed for their production. These data served as a basis for planning and programming of purchases of materials and production of products, from the release of the planned orders, the orders receipts, inventory control, to monitor the weekly product demand, thus contributing for a solid and optimized production process. They were obtained as results the decrease in raw material inventories, increased productivity by reducing production downtime due to lack of materials and the reduction in delays of deliveries of production orders.
\end{abstract}

Key-words: Inventory management, Material Requirement Planning, MRP, Production.

\title{
Referências
}

ARNOLD, J. R. T. Administração de materiais: uma introdução. $2^{a}$ Edição: São Paulo: Atlas, 2012. 
CHIROLI, D.M.G. Logística de produção e serviços. Reimpressão revista e atualizada. Maringá: Editora UniCesumar, 2014.

CORRÊA, H.L.; GIANESI, I.G.N.; CAON, M. Planejamento, Programação e Controle da Produção. $4^{\mathrm{a}}$ Edição. São Paulo: Editora Atlas, 2001.

CORRÊA, H.L.; GIANESI, I.G.N.; CAON, M. Planejamento, Programação e Controle da Produção. $5^{\mathrm{a}}$ Edição. São Paulo: Editora Atlas, 2008.

DIAS, M.A.P. Administração de materiais: uma abordagem logística. 5 Edição: São Paulo: Atlas, 2010.

GIL, A.C. Métodos e técnicas de pesquisa social. 6ª Edição. São Paulo: Editora Atlas, 2008.

POTER, M.E. Competição: Estratégias competitivas essenciais. Tradução por: Afonso celso da Cunha Serra. Rio de janeiro, Campus. 1999.

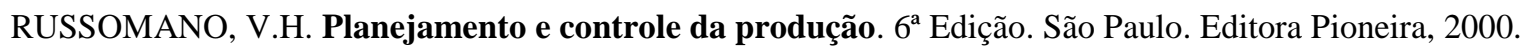

SILVA, E.L.; MENEZES, E.M. Metodologia da pesquisa e elaboração da dissertação. $4^{\mathrm{a}}$ Edição. Florianópolis: UFSC, 2005.

SLACK, N.; JOHNSTON, R.; CHAMBERS, S.; HARLAND, C.; HARRISON, A. Administração da Produção. Edição compacta: Atlas, 1999

TUBINO, D.F. Manual de planejamento e controle da produção. 2a Edição. São Paulo. Editora Atlas, 2000.

TUBINO, D.F. Planejamento e controle da produção: teoria e prática. São Paulo. Editora Atlas, 2007.

\section{Dados do autores:}

Nome completo: Daiane Maria De Genaro Chiroli

Doutora em Engenharia de Produção pela UFSC

Filiação institucional: Universidade Estadual de Maringá

Departamento: Engenharia de Produção

Função ou cargo ocupado: Professor Adjunto

Endereço completo para correspondência: Av. Colombo, 5790 Jd. Universitário Maringá-Pr-Brasil

CEP 87020-900

Telefones para contato: 44-3011-5819

E-mail:dmgenaro@hotmail.com

Nome completo: Karen de Oliveira Valério

Graduada em Engenharia de Produção pela UEM

Filiação institucional: Riachuelo

Função ou cargo ocupado: Trainee Engenharia de Produção

Endereço completo para correspondência: Av. Colombo, 5790 Jd. Universitário Maringá-Pr-Brasil CEP 87020-900

Telefones para contato: 44-3011-5819

E-mail: karen.valerio@yahoo.com.br

Submetido em: 30-03-2016

Aceito em: 15-06-2016 